\title{
Review of: "Community structure and diversity characteristics of rhizosphere and root endophytic bacterial community in different Acacia species"
}

Dinesh Raghavan

Potential competing interests: The author(s) declared that no potential competing interests exist.

Community structure and diversity characteristics of rhizosphere and root endophytic bacterial community in different Acacia species

Zong-sheng Yuan, Fang Liu, Shi-bin He, Li-li Zhou, Hui Pan

The study provides an analysis of the diversity of rhizosphere and root endophytic bacterial communities in different Acacia species. This is an interesting study and provides insights into the role of rhizosphere and root endophytic bacterial communities in different Acacia species.

For this purpose, samples of roots, rhizosphere soil, and non-rhizosphere soil were collected from five Acacia species: Acacia crassicarpa, A. Cunn. ex. Benth, Acacia cincinnata F. Muell., Acacia melanoxylon R.Br., Acacia mangium Willd., and Acacia mearnsil De Wild. Corresponding samples from E. urophylla $\times$ E. grandis trees in the same area were used as control.

No scientific explanation on how these trees were selected for sampling is provided, though the authors state that the trees were randomly selected. A brief description on the history of these trees like age, soil type in the area and basic soil properties could have been provided.

Were the trees of the same age? What was the soil type? was the soil type similar at all the sampling sites? what was the land area covered during random selection of these trees? These are some points missing in the paper.

Proteobacteria, followed by Actinobacteria and Acidobacteria, were identified as the dominant bacteria in the root endophytic bacterial population. In the soil samples, Acidobacteria was the most abundant, followed by Proteobacteria and Actinobacteria. The authors suggest that this variation in core soil bacteria is mainly due to $\mathrm{N}$ fixing nature of the tree species. However, no data is provided to validate this claim. Also, explanation on intra-tree species variation (within Acacia species) in soil bacterial communities could have been more clearly elucidated, while variation between soil, non-rhizosphere and rhizosphere samples is expected and clearly explained. 
The functions of specific bacterial communities, is predicted using PICRUSt. But not much is provided by way of explaining the variations in the metabolic functions between treatments.

What was the PICRUSt version used? Was Green genes DB used as reference while performing functional analysis using PICRUST?

These are just a few points that could have been included in the paper.

Still, the paper is an interesting read.

I will give it $3.5^{*}$. 